\title{
Network Reconfiguration for an Electric Distribution System with Distributed Generators based on Symbiotic Organisms Search
}

\author{
Thanh Long Duong \\ Faculty of Electrical Engineering Technology \\ Industrial University of Ho Chi Minh City \\ Ho Chi Minh City, Vietnam \\ duongthanhlong@iuh.edu.vn
}

\author{
Thuan Thanh Nguyen \\ Faculty of Electrical Engineering Technology \\ Industrial University of Ho Chi Minh City \\ Ho Chi Minh City, Viet Nam \\ nguyenthanhthuan@iuh.edu.vn
}

\begin{abstract}
This paper proposes a method of network reconfiguration based on symbiotic organisms search (SOS) algorithm for reducing power loss of the electric distribution system. The SOS is a recent developed meta-heuristic algorithm inspired from the symbiotic interaction strategies of organisms for surviving and propagating in the ecosystem. Compared to other algorithms, SOS does not need any control parameters during the searching process. The advantages of the proposed SOS method have been validated in two electric distribution systems. Three network cases have been considered for each system, consisting of performing network reconfiguration on the system without distributed generator (DG) placement, the system installed type-P DGs and the system installed type-PQ DGs. The comparison results with particle swarm optimization and other previous methods show that the proposed SOS can be a promising technique for the problem of electric network reconfiguration.
\end{abstract}

Keywords-symbiotic organisms search; electric distribution system; network reconfiguration; active power loss

\section{INTRODUCTION}

Power loss in electric distribution systems (EDSs) takes a high portion in the total power loss of the electricity system. There are lots of techniques for reducing power loss such as distributed generation placement [1], capacitor placement [2-4], and network reconfiguration. Among these methods, network reconfiguration is the most efficient. It is implemented by transferring a part of the load from some branches to others through changing the status of switches located on each branch. The result of network reconfiguration method is a radial topology of EDS that satisfy constraints such as the limits of node voltage and branch current. From its introduction [5], the problem of network reconfiguration has been solved by many methods. They can be divided into two main groups: heuristic and metaheuristic. The first main group includes methods based on knowledge of operating the EDN and technical strategies. Typical methods in this group are grouped in branch and bound methods and branch exchange methods. The idea of branch and bound method is that the EDN is modeled by a purely resistive network. Initially all switches are closed. Gradually the switches on the branches with the smallest currents are opened step by step until the radial topology of the EDN is reached [6]. In the branch exchange method, initially all switches are closed and then an open switch is replaced by a closed switch for reducing power loss [7]. The advantages of this method group are that they are easy to implement and the knowledge related to these methods is very close to the area of operating EDS. However, the obtained results are usually local extremes. In addition, changing the objective function is difficult for solving the problem of network reconfiguration. The second group method includes methods based on metaheuristic algorithms. They are usually inspired from phenomena of nature or society. The main advantage of this method group is that they are very efficient for handling constraints and different objective functions. Compared to the former group, the later has the ability to provide a sufficiently good solution to the problem of network reconfiguration. Thus, more and more new metaheuristic algorithms have being applied to this problem such as genetic algorithm [8], particle swarm optimization (PSO) algorithm [9-11], gravitational search algorithm (GSA) [12], fireworks algorithm (FWA) [13], runner root algorithm (RRA) [14, 15], and cuckoo search algorithm (CSA) [16, 17]. However, for solving the problem of network reconfiguration by this method group, the issue of adjusting the control parameters of the algorithms has to be faced, e.g. for applying the GA to the problem of network reconfiguration, the rates of selection and mutation have to be tuned, for applying the PSO, two constants $C_{1}$ and $C_{2}$ in the equation of velocity of particles have to be selected etc. Therefore, it is worth considering the application of recent developed metaheuristic algorithms which have less control parameters, for solving the network reconfiguration problem.

Symbiotic organisms search (SOS) is inspired from the symbiotic interaction strategies of organisms for surviving and propagating in the ecosystem [18]. Compared to other metaheuristic algorithms, the biggest advantages of SOS are that it does not require specific algorithmic parameters for operations. In SOS, from each organism in the ecosystem, four new organisms are generated based on mutualism, commensalism, and parasitism phases. Two new organisms are used for updating the initial organism and the other two 
organisms are used for updating the other organisms in the ecosystem. In [18], SOS has been used to solve 26 benchmark functions and has outperformed other algorithms such as GA, PSO, etc. Recently, SOS has been applied in many areas such as construction engineering, power or energy optimization engineering problems [19]. In the latter, SOS has been successful applied in the problems of optimal placement and sizing of distributed generators (DGs) [20, 21], reduction of power loss of a transmission system [22], capacitor placement [23], economic emission dispatch problem [24], and optimal power flow [25]. For the problem of network reconfiguration, SOS has been used in [26]. However, in this study, the solution vector of SOS (called SOS1) for the problem is expressed by the state of all the electric switches in the EDS. The normally closed electric switches are shown by the one state and vice versa, the open electric switches are represented by the zero state. Coding technique makes the solution vector become large and makes SOS ineffective for finding optimal network configuration. In this paper, the proposed method based on SOS is used to solve the problem of network reconfiguration to find the optimum EDS configuration for power loss reduction. The solution vector of the problem is only presented by the open switches in integer form. The applied method was tested on the 33-bus and 69-bus systems and the obtained results were compared with the ones of other techniques. The main contributions of this paper can be summarized as:

- SOS is adapted to solve efficiently the network reconfiguration problem.

- The performance of SOS is effectively applied on different electric distribution systems, namely the EDS without DGs and with different types of DGs.

- SOS can find the optimal radial topology of EDS for all cases, with higher success rate than the other methods mentioned in the literature.

\section{THE PROBLEM OF NETWORK RECONFIGURATION}

The power loss of EDS is determined by the sum of the power losses of each branch of the system. Although having mesh structure, EDSs always operate in radial topology. So, there are branches that contain switches in the open state with no current flow. Therefore, the total power loss of the EDS is determined by:

$$
\Delta P=\sum_{i=1}^{N b r} k_{i} R_{i} \frac{P_{i}^{2}+Q_{i}^{2}}{V_{i}^{2}}
$$

where $N b r$ is a number of branches of the EDS, $R_{i}$ is the $i$-th branch's resistance, $P_{i}$ and $Q_{i}$ are respectively the active and reactive power flow on the $i$-th branch. $k_{i}$ is a coefficient which is equal to zero for an open branch and one for a close branch, and $V_{i}$ is the ending voltage of the $i$-th branch. Equation (1) is subjected to the following constraints:

- Radial network constraint: The radial topology of EDS must be maintained and no load nodes have not to be served. In this study, the empirical formula from [27] is used to check whether the candidate configuration is radial or not:

$$
\operatorname{det}(A)= \begin{cases}-1 \text { or } 1, & \text { radial } \\ 0, & \text { not radial }\end{cases}
$$

where $\operatorname{det}(A)$ is the determinant of matrix $A$. $A$ is a (branch $x$ node) matrix presenting a connection of EDS.

- Node voltage constraint: The voltage magnitude at each node must lie in the permissible range $\left[V_{\min }, V_{\max }\right]$ to keep power quality. In general, $V_{\min }, V_{\max }$ are usually set equal to $[0.95,1] \mathrm{pu}$ :

$$
V_{\text {min }} \leq V_{j} \leq V_{\max } \text { with } j=1,2, . . \text { Nbus }
$$

where Nbus is the total number of nodes in EDS.

- Branch current constraint: The current magnitude of each branch of EDS must lie in their permissible range to avoid over load:

$$
I_{i} \leq I_{\max , i} \text { with } i=1,2, \ldots N b r
$$

\section{SOS FOR NETWORK RECONFIGURATION}

SOS is based on the interactive behavior of organisms in nature. Many species living in nature have a reliant relationship with other species, called symbiosis. Commonly, there are three popular reliant relationships in nature, mutualism, commensalism, and parasitism [18-19]. The main procedures of SOS for the problem of network reconfiguration problem are described below.

\section{A. Initializating Candidate Solutions}

Similarly to other metaheuristic algorithms based on population, SOS also generates an initial population of organisms termed as the ecosystem. Each organism represents a candidate solution of the optimization problem. The degree of adaptation of each organism in the ecosystem is reflected by its fitness value. The process of creating the initial population of organisms is implemented as:

$$
X_{i}=X_{\text {low }, k}+\text { rand } \times\left(X_{\text {high }, k}-X_{\text {low }, j}\right)
$$

where $X_{i}(i=1,2 \ldots \mathrm{N})$ is an organism in the ecosystem. $X_{\text {high }, k}$ and $X_{\text {low }, k}$ are the upper and lower limits of the control variable $k(\mathrm{k}=1,2, \ldots, D)$ of the organism, $N$ and $D$ are the population size and the dimension of the problem.

The control variables of the network reconfiguration problem are the switches located in each branch of the EDS. Therefore, the control variables used in SOS for the network reconfiguration problem are presented under integer form. In addition, the search space of each control variable is also determined by closing the corresponding initial open switch $X_{\text {initial }, k}$. By closing $X_{\text {initial }, k}$, a loop occurs in the EDS called $\operatorname{Loop}_{k}$. All the switches in this loop are candidate open switches for the control variable $X_{i, k}$. It is clear that the size of $\operatorname{Loop}_{k}$ is the upper limit of the control variable $X_{i, k}$ and the lower limit of the control variable $X_{i, k}$ is one, which is the first position in Loop $_{k}$. In other words the control variables of the network reconfiguration problem are the position of switches in each loop created by closing one by one the initial open switches in the EDS. Therefore, in the initialization state, the ecosystem is generated as:

$$
X_{i}=\operatorname{round}\left[X_{\text {low }, k}+\operatorname{rand} \times\left(X_{\text {high }, k}-X_{\text {low }, j}\right)\right]
$$


where round is a function that rounds each element of $X_{i}$ to the nearest integer.

From the initial population of organisms, the radial topology of each organism is checked and if it is satisfied, the power flow is run to calculate its fitness function. Then, based on the fitness value, the best organism $X_{\text {best }}$ of the ecosystem is found. If the radial topology of organism $X_{i}$ is not satisfied, a very high value is assigned to its fitness function value.

\section{B. Generating New Solutions Based on Mutualism Strategy}

In nature, mutualism helps in gaining benefits for both participating organisms in the ecosystem. An example of this strategy can be seen from the relationship between flowers and bees. From this metaphor, the organism $X_{i}$ in the population will interact with the organism $X_{j}$ which is selected randomly from the population to create two new solutions as in (7). Participating in the mutualism strategy, both organisms gain benefits. The radial topology of the two new organisms is checked and if it is satisfied, the power flow is run to calculate their fitness function. Then, if their fitness values are better than the one before interaction, the organisms $X_{i}$ and $X_{j}$ will be updated:

$$
\left\{\begin{array}{l}
X_{i, \text { new }}=\operatorname{round}\left[X_{i}+\operatorname{rand}(0,1) \times\left(X_{\text {best }}-M_{\text {vector }} \times B F 1\right)\right] \\
X_{j, \text { new }}=\operatorname{round}\left[X_{j}+\operatorname{rand}(0,1) \times\left(X_{\text {best }}-M_{\text {vector }} \times B F 2\right)\right]
\end{array}(7)\right.
$$

$M_{\text {vector }}$ is determined by (8). $B F 1$ and $B F 2$ are the benefit factors representing for level of benefit of each organism determined by:

$$
\begin{gathered}
M_{\text {vector }}=\frac{X_{i}+X_{j}}{2} \\
\left\{\begin{array}{l}
B F 1=\text { round }\left(1+\operatorname{rand}_{1}\right) \\
B F 2=\text { round }\left(1+\operatorname{rand}_{2}\right)
\end{array}\right.
\end{gathered}
$$

\section{Generating New Solutions with Commensalism Strategy}

In nature, many birds live on bigger herbivores. This is a typical example of commensalism. In this strategy, a species will benefit while the other derives neither benefit nor harm. The organism $X_{i}$ will benefit from the commensal interaction between organism $X_{i}$ and $X_{j}$. To implement this strategy, organism $X_{j}$ is selected randomly from the ecosystem to interact with $X_{j}$ as presented in (10) for creating a new organism. Then, organism $X_{i}$ will be updated only if the fitness of the new organism is better than that of $X_{i}$ before the interaction.

$$
X_{i, \text { new }}=\operatorname{round}\left[X_{i}+\operatorname{rand}(-1,1) \times\left(X_{\text {best }}-X_{j}\right)\right]
$$

\section{Generating New Solutions with Parasitism Strategy}

Contrary to commensalism strategy, in the parasitism one species will benefit while the other species as a host will be a victim and even may die. In SOS, organism $X_{i}$ is considered as a parasite. It modifies randomly some control variables to create a new organism called $X_{\text {Parasite }}$. Therefore, $X_{\text {Parasite }}$ is first assigned by $X_{i}$, and then some of the control variables of $X_{\text {Parasite }}$ are modified by (11). Note that the number of control variables of $X_{\text {Parasite }}$ is also selected randomly. The organism $X_{j}$ which is considered a host is selected randomly. If the fitness function value of the new organism created from $X_{i}$ is better than that of the organism $X_{j}$, it will replace the position of $X_{j}$ in the ecosystem. In other words, $X_{j}$ will be killed by $X_{i}$.

$$
X_{\text {Parasite }, k}=\operatorname{round}\left[X_{\text {low }, k}+\operatorname{rand} \times\left(X_{\text {high }, k}-X_{\text {low }, k}\right)\right]
$$

After performing mutualism, commensalism, and parasitism for the organism $X_{i}$, the best organism is updated at the end. Then, the next organism in the ecosystem is considered until the whole population interacts with the three strategies. The process of searching new solution is stopped if the maximum number of organism evaluations is reached. The flowchart of the proposed SOS for the network reconfiguration problem is given in Figure 1.

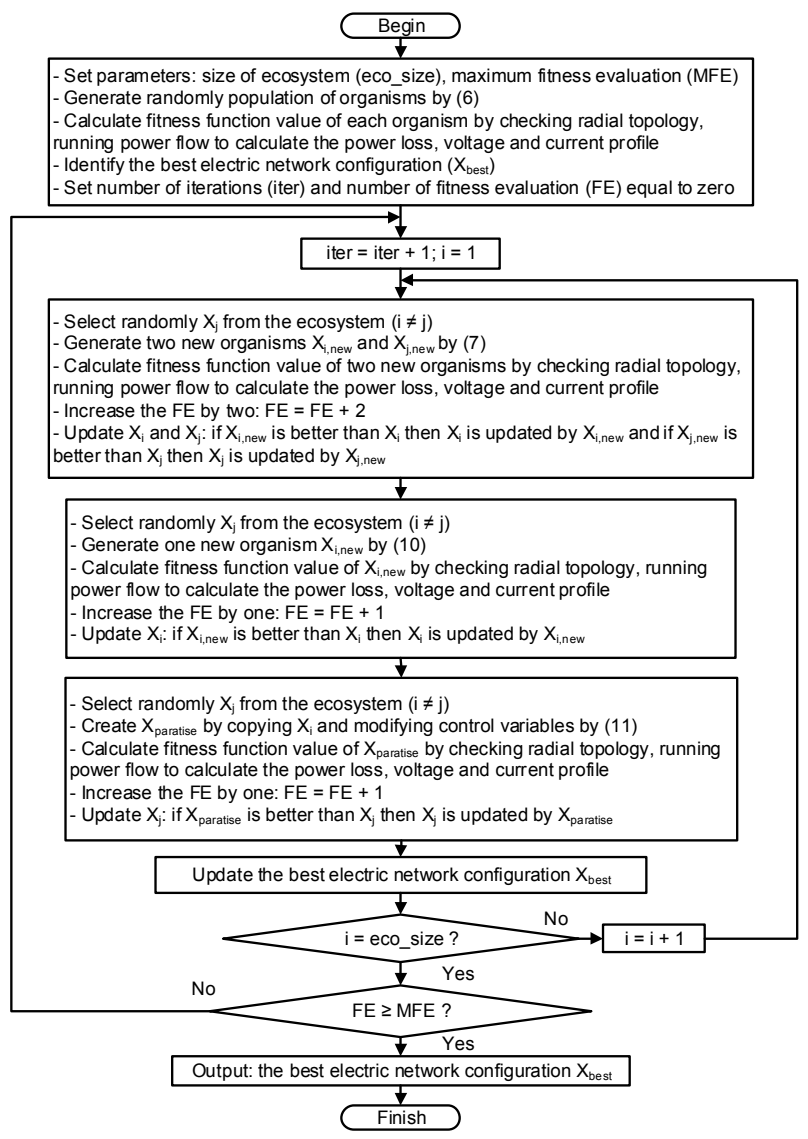

Fig. 1. SOS for the network reconfiguration problem

\section{NUMERICAL RESULTS}

To demonstrate the efficiency of the proposed method, the application of SOS for network reconfiguration is implemented in Matlab 2016a, executed on a personal computer Intel(R) Core(TM) i5-2430 at $2.4 \mathrm{GHz}$ with $4 \mathrm{~GB}$ RAM. The performance of SOS is evaluated in a 33-node and a 69-node system. Also, network reconfiguration based on the wellknown PSO algorithm was implemented on the same personal computer in order to compare its results with the proposed method's. 


\section{A. The 33-node Test System}

The $12.66 \mathrm{kV}, 33$-node test system contains 1 feeder and 32 load nodes. The system's data are taken from [28]. The five initial open switches are $\{\mathrm{s} 33, \mathrm{~s} 34, \mathrm{~s} 35, \mathrm{~s} 36$ and s37\} causing power loss of $202.6863 \mathrm{~kW}$. The diagram of the system for the initial configuration is given in Figure 2. The control parameters of SOS and PSO consisting of eco_size, pop_size and MFE are set to 10, 40 and 2000 respectively.

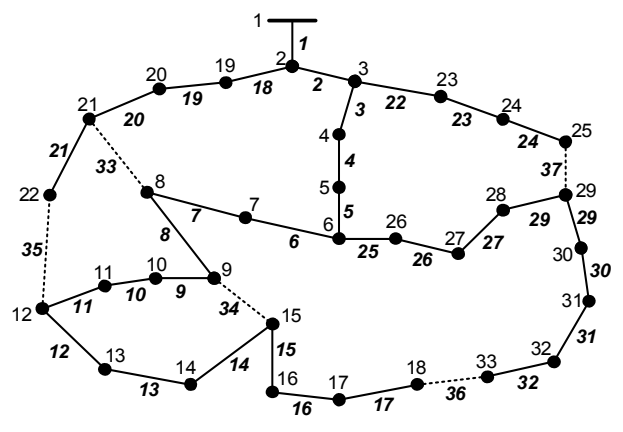

Fig. 2. The 33-node test system

Three cases were considered for the 33-node test system:

- Case-1: Performing network reconfiguration on the system without DG placement.

- Case-2: Performing network reconfiguration on the system with installed type-P DGs which inject only real power such as fuel and photovoltaic cells.

- Case-3: Performing network reconfiguration on the system with installed type-PQ DGs which inject real and reactive power like synchronous machines.

The calculated results on the 33-node test system for the case- 1 are shown in Table I. The total power loss $(\Delta P)$ of the system has been reduced from $202.6863 \mathrm{~kW}$ to $139.9823 \mathrm{~kW}$ by applying the proposed SOS method. This minimal power loss value is achieved by opening switches $\{\mathrm{s} 7, \mathrm{~s} 9, \mathrm{~s} 14, \mathrm{~s} 28, \mathrm{~s} 32\}$. The minimal voltage amplitude $\left(V_{\min }\right)$ has been raised from 0.9131 p.u. before reconfiguration to 0.94124 p.u. after SOS reconfiguration. In comparison with PSO, in case- 1 , PSO found the optimal configuration but in 50 runs PSO has only achieved the optimal solution 5 times corresponding to $10 \%$, while SOS achieved the optimal configuration 38 times corresponding to $76 \%$. The maximal $\left(F i t_{\max }\right)$, average $\left(F i t_{\min }\right)$ and STD of the fitness function values (fit) of SOS were also lower than those of PSO in 50 runs. Furthermore, the running time of SOS was 1.2228 s lower than PSO's. The average, minimal and maximal convergence trends of SOS and PSO in 50 runs for case- 1 are shown in Figure 3. In 50 runs, the average convergence trend of SOS is much closer to the minimal convergence trend than of PSO. This means that in each run, SOS usually tends to convergence to lower fitness values than of PSO. As shown in Table II, the results achieved by SOS are identical to those obtained by improved selective binary PSO (ISBPSO) [29] and FWA [13]. Regarding the comparison with RRA [14], power loss obtained by SOS is 0.4323 higher than by RRA, however the minimal voltage amplitude of network configuration obtained by SOS is more improved than by RRA. Table II shows that the network configuration obtained by SOS1 does not satisfy the radial topology due to the existing ten open switches of $\{22,23,24,30,31,32,33,34,35,37\}$ in the optimal solution. This configuration will make some nodes in the system not supplied with power. The power loss obtained by SOS1 is $175.3337 \mathrm{~kW}$ which is also higher than that of the proposed SOS.

TABLE I. RESULTS OF CASE-1 OF THE 33-NODE SY STEM AFTER 50 INDEPENDENT RUNS

\begin{tabular}{|c|c|c|c|}
\hline Item & Initial & SOS & PSO \\
\hline \multirow{2}{*}{ Opened switches } & 33,34, & $7,9,14$, & $7,9,14,28$, \\
& $35,36,37$ & 28,32 & 32 \\
\hline$\Delta \boldsymbol{P}(\mathbf{k W})$ & 202.6863 & 139.9823 & 139.9823 \\
\hline $\boldsymbol{V}_{\boldsymbol{m i n}}$ (p.u.) & 0.9131 & 0.94124 & 0.94124 \\
\hline Fit $_{\boldsymbol{m a x}}$ & - & 159.5083 & 194.1428 \\
\hline Fit $_{\text {min }}$ & - & 148.7392 & 148.7392 \\
\hline Fit $_{\text {average }}$ & - & 150.2977 & 161.4816 \\
\hline STD of fit. & - & 3.0475 & 8.2984 \\
\hline Successful rate (\%) & - & 76 & 10 \\
\hline run time (sec) & - & 4.3819 & 5.6047 \\
\hline
\end{tabular}

TABLE II. COMPARISON OF SOS WITH OTHER METHODS IN THE 33NODE SYSTEM FOR CASE-1

\begin{tabular}{|c|c|c|c|}
\hline Method & Opened switches & $\Delta \boldsymbol{P}(\mathbf{k W})$ & $\boldsymbol{V}_{\boldsymbol{m i n}}$ (p.u.) \\
\hline SOS & $7,9,14,28,32$ & 139.9823 & 0.94124 \\
\hline SOS1 [26] & $\begin{array}{c}22,23,24,30,31, \\
32,33,34,35,37\end{array}$ & 175.3337 & - \\
\hline ISBPSO [29] & $7,14,9,32,37$ & 139.55 & 0.9378 \\
\hline CSA [17] & $7,9,14,28,32$ & 139.9823 & 0.94124 \\
\hline RRA [14] & $7,14,9,32,37$ & 139.55 & 0.9378 \\
\hline FWA [13] & $7,9,14,28,32$ & 139.9823 & 0.94124 \\
\hline
\end{tabular}

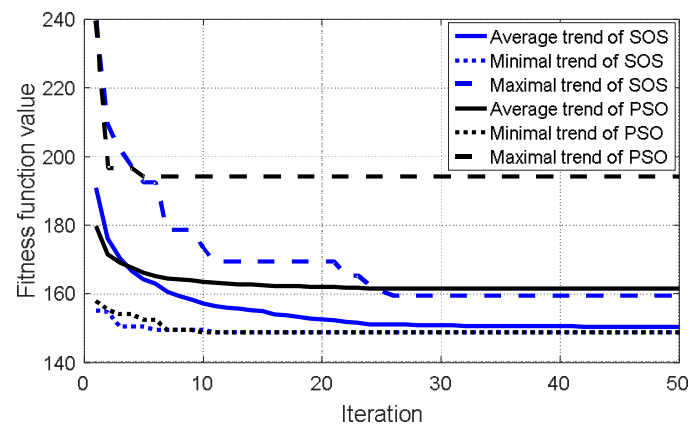

Fig. 3. Convergence trends of SOS and PSO in 50 runs for the 33-node test system in case- 1

In the case of the test system with three type-P DGs installed with sizes of $0.7798 \mathrm{MW}, 1.1251 \mathrm{MW}$ and $1.3496 \mathrm{MW}$ at node 14,24 and 30 . The reconfiguration results are presented in Table III. The power loss has been reduced from $74.2576 \mathrm{~kW}$ of the initial configuration to $61.4474 \mathrm{~kW}$ after the reconfiguration with the opening of $\{\mathrm{s} 7, \mathrm{~s} 8, \mathrm{~s} 9, \mathrm{~s} 36$ and $\mathrm{s} 37\}$ switches. The obtained result from SOS is $1.5326 \mathrm{~kW}$ lower than the one obtained from CSA. In this case, PSO also found the optimal configuration but in 50 runs, PSO only achieved the optimal solution 3 times corresponding to $6 \%$. Meanwhile, SOS achieved the optimal configuration 28 times corresponding to $56 \%$. Similarly, the maximal, average and STD of the fitness function values of SOS were also lower than 
those of PSO in 50 runs. Furthermore, the running time of SOS was 1.3612s lower than PSO's. The convergence trends of proposed SOS and PSO in 50 runs for case- 2 are shown in Figure 4.

TABLE III. RESULTS OF CASE-2 OF THE 33-NODE SY STEM AFTER 50 INDEPENDENT RUNS

\begin{tabular}{|c|c|c|c|c|}
\hline Item & Initial & SOS & PSO & CSA [17] \\
\hline Opened switches & $\begin{array}{c}33,34,35, \\
36,37\end{array}$ & $\begin{array}{c}7,8,9, \\
36,37\end{array}$ & $\begin{array}{c}7,8,9, \\
36,37\end{array}$ & $\begin{array}{c}8,9,27, \\
33,36\end{array}$ \\
\hline $\begin{array}{c}\text { DG size in MW } \\
\text { (node) }\end{array}$ & \multicolumn{4}{|c|}{$0.7798(14) ; 1.1251$} \\
\hline $\boldsymbol{P}_{\text {(kW) }}$ & $74.257) ; 1.3496(30)$ \\
\hline $\boldsymbol{V}_{\min }$ (p.u.) & 0.9778 & 0.97865 & 0.97865 & 0.9826 \\
\hline Fit $_{\text {max }}$ & - & 61.9765 & 63.328 & - \\
\hline Fit $_{\min }$ & - & 61.4474 & 61.4474 & - \\
\hline Fit $_{\text {average }}$ & - & 61.503 & 62.007 & - \\
\hline STD value of fit. & - & 0.1015 & 0.3742 & - \\
\hline $\begin{array}{c}\text { Successful rate } \\
\text { (\%) }\end{array}$ & - & 56 & 6 & - \\
\hline $\begin{array}{c}\text { Running time } \\
\text { (sec) }\end{array}$ & - & 3.9691 & 5.3303 & - \\
\hline
\end{tabular}

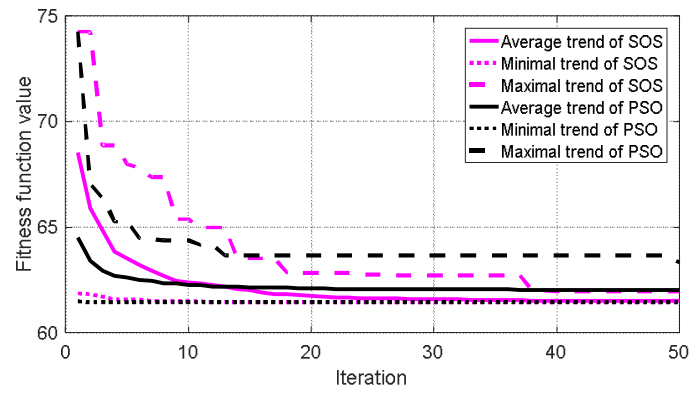

Fig. 4. Convergence trends of SOS and PSO in 50 runs for the 33-node test system in case-2

Similarly to case-2, the comparison of SOS and PSO in case- 3 shown in Table IV also leads to the conclusion that SOS is better than PSO in terms of successful rate, quality of fitness function values, and running time. For the network with three type-PQ DGs with sizes of $0.5314+\mathrm{j} 0.3147 \mathrm{MW}$, $0.5030+\mathrm{j} 0.1485 \mathrm{MW}, 1.0403+\mathrm{j} 0.9996 \mathrm{MW}$ at node 12,16 and 30 , after reconfiguration SOS gained the optimal configuration in 32 runs corresponding to $64 \%$ while PSO did it only in 2 runs. The maximum, average and STD of the fitness function values of SOS were respectively $19.635,18.9885$ and 0.2518 while they were $21.3489,19.5861$ and 0.4703 for PSO. Moreover, the running time of SOS was 1.3668 s lower than PSO's. The average, minimum and maximum convergence trends of proposed SOS and PSO in 50 runs for case- 1 are shown in Figure 5. The power loss of the optimal configuration obtained by SOS was also 6.3120 lower than that of the method based on grey wolf optimizer (GWO) [30]. The voltage profile of the system achieved by the proposed SOS technique is shown in Figure 6. In the initial configuration there are many nodes with lower voltage magnitude than the permissible range such as node 6-18 and 27-33. Most of them have been improved after the reconfiguration by SOS in case-1. Only nodes $18,30-33$ have voltage magnitude lower than 0.95 p.u. In case- 2 and case- 3 , the voltage magnitudes of all the nodes are within the permissible range. The current profile of the 33- node system before and after reconfiguration is shown in Figure 7. From the Figure we can see that the currents of all branches are within the permissible range and the branches carrying high load such as branches 1 to 5 have reduced loads after the reconfiguration.

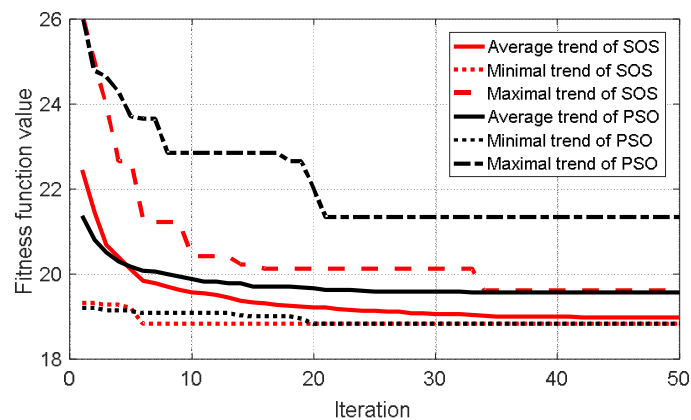

Fig. 5. Convergence trends of SOS and PSO in 50 runs for the 33-node test system in case- 3

TABLE IV. RESULTS OF CASE-3 OF THE 33-NODE SY STEM AFTER 50 INDEPENDENT RUNS

\begin{tabular}{|c|c|c|c|c|}
\hline Item & Initial & SOS & PSO & GWO [30] \\
\hline Opened switches & $\begin{array}{c}33,34 \\
35,36,37\end{array}$ & $\begin{array}{l}6,14,21 \\
28,32\end{array}$ & $\begin{array}{l}6,14,21 \\
28,32\end{array}$ & $\begin{array}{c}7,9,14,32, \\
37\end{array}$ \\
\hline $\begin{array}{l}\text { DG size in MVA } \\
\text { (node) }\end{array}$ & \multicolumn{4}{|c|}{$\begin{array}{c}0.5314+\mathrm{j} 0.3147(12) ; 0.5030+\mathrm{j} 0.1485(16) \\
1.0403+\mathrm{j} 0.9996(30)\end{array}$} \\
\hline$\Delta P(\mathbf{k W})$ & 26.0931 & 18.8366 & 18.8366 & 25.1486 \\
\hline$V_{\min }$ (p.u.) & 0.9807 & 0.98638 & 0.98638 & - \\
\hline Fit $_{\max }$ & - & 19.635 & 21.3489 & - \\
\hline Fit $_{\text {min }}$ & - & 18.8366 & 18.8366 & - \\
\hline Fit $_{\text {average }}$ & - & 18.9885 & 19.5861 & - \\
\hline STD of fit. & - & 0.2518 & 0.4703 & - \\
\hline Successful rate (\%) & - & 64 & 4 & - \\
\hline Run time (sec) & - & 4.0591 & 5.4259 & - \\
\hline
\end{tabular}

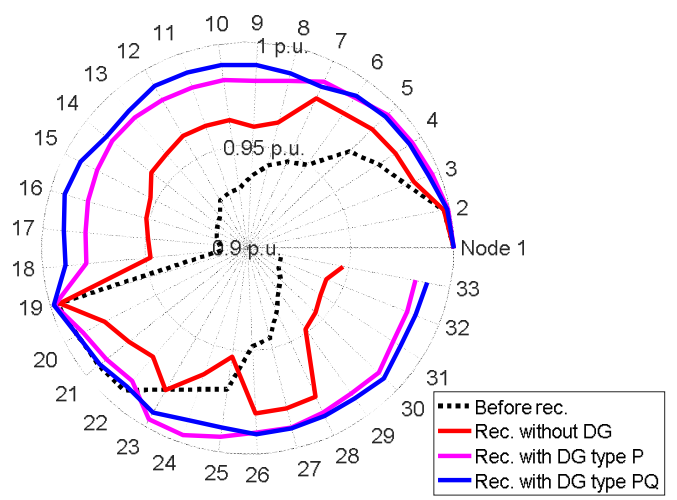

Fig. 6. Voltage profile of the 33-node test system before and after reconfiguration

\section{B. The 69-node Test System}

The $12.66 \mathrm{kV}, 69$-node test system contains 1 feeder and 68 load nodes. The system's data are taken from [31]. The five initially open switches $\{\mathrm{s} 69, \mathrm{~s} 70, \mathrm{~s} 71, \mathrm{~s} 72$ and s73\} caused power loss of $224.8871 \mathrm{~kW}$. The diagram of the system for the initial configuration is given in Figure 8. The control parameters of SOS and PSO selected for the 69-node test system are similar to those of the 33-node test system. As in the 
33-node test system, the three aforementioned cases were also considered.

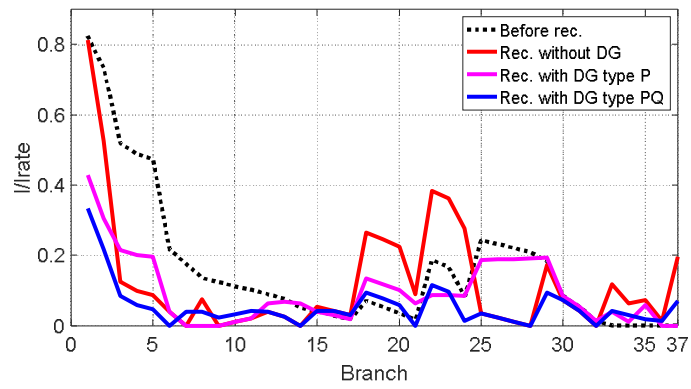

Fig. 7. Current profile of the 33-node test system before and after performing reconfiguration

The simulation results on the 69-node test system for case-1 are shown in Table V. The total power loss of the system has been reduced from $224.8871 \mathrm{~kW}$ to $98.5875 \mathrm{~kW}$ after performing reconfiguration. This minimal power loss value is achieved by opening switches $\{\mathrm{s} 14, \mathrm{~s} 57, \mathrm{~s} 61, \mathrm{~s} 69$ and s70\}. In comparison with PSO, in case-1, PSO achieved the optimal solution 12 times in 50 rounds corresponding to $24 \%$ while SOS achieved the optimal configuration 23 times corresponding to $46 \%$. The maximum, average and STD of the fitness function values of SOS were lower than those of PSO in 50 runs. Furthermore, the running time of SOS was 2.421s lower than PSO's. The average, minimal and maximal convergence trends of the proposed SOS and PSO in 50 runs for case-1 are shown in Figure 9. As shown in the Figure, in 50 runs the average convergence trend of SOS converged to lower values than PSO and was much closer to the minimal convergence trend than PSO. As presented in Table VI, the results achieved by SOS are identical to those obtained by CSA [17]. In comparison with FWA [32] and adaptive shuffled frogs leaping algorithm (ASFLA) [33], the power loss obtained by SOS is the same with that of FWA and ASFLA.

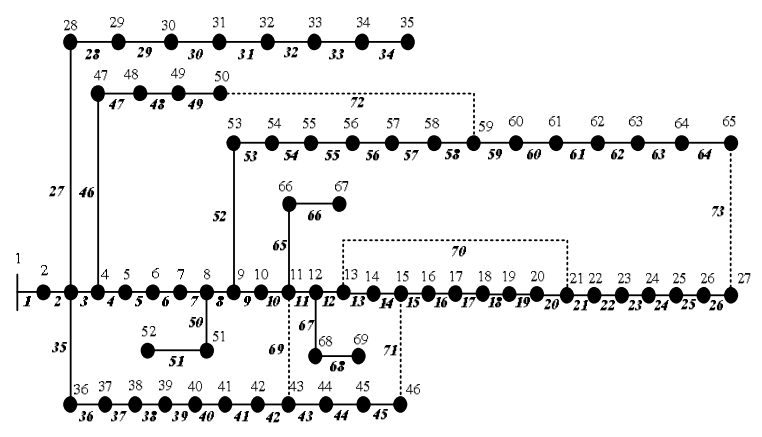

Fig. 8. The 69-node test system

In the case of the test system with three type-P DGs installed with sizes of $0.6022,0.3804$ and $2 \mathrm{MW}$ at node 11,18 and 61 , the reconfiguration results are presented in Table VII. The power loss has been reduced from $72.4343 \mathrm{~kW}$ of the initial configuration to $41.1317 \mathrm{~kW}$ after reconfiguration with the opening of $\{s 14, s 57, s 64, s 69$ and s70 $\}$ switches. The power loss obtained from SOS is identical to that obtained from CSA.
PSO also found the optimal configuration but achieved it only 2 times in 50 runs. Meanwhile SOS achieved the optimal configuration 21 times corresponding to $42 \%$. Similarly, the maximal, average, and STD of the fitness function values of SOS were lower than those of PSO. Furthermore, the running time of SOS was 2.7578 s lower than PSO's. The convergence trends of proposed SOS and PSO in 50 runs for the case- 2 are shown in Figure 10.

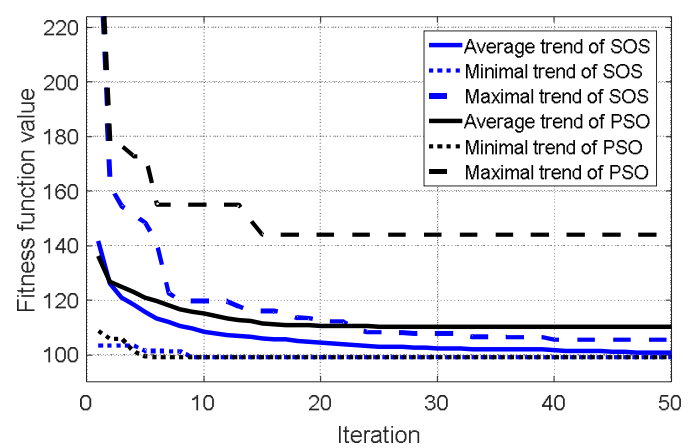

Fig. 9. Convergence trends of SOS and PSO in 50 runs for the 69-node test system in case-1

TABLE V. RESULTS OF CASE-1 OF THE 69-NODE SY STEM AFTER 50 INDEPENDENT RUNS

\begin{tabular}{|c|c|c|c|}
\hline Item & Initial & SOS & PSO \\
\hline \multirow{2}{*}{ Opened switches } & $\begin{array}{c}69,70,71, \\
72,73\end{array}$ & $\begin{array}{c}14,57,61, \\
69,70\end{array}$ & $\begin{array}{c}14,57,61, \\
69,70\end{array}$ \\
\hline$\Delta \boldsymbol{P}(\mathbf{k W})$ & 224.8871 & 98.5875 & 98.5875 \\
\hline $\boldsymbol{V}_{\min }$ (p.u.) & 0.9092 & 0.9495 & 0.9495 \\
\hline Fit $_{\max }$ & - & 105.6075 & 143.9785 \\
\hline Fit $_{\text {min }}$ & - & 99.1169 & 99.1169 \\
\hline Fit $_{\text {average }}$ & - & 100.7601 & 110.2838 \\
\hline STD of fit. & - & 2.5010 & 13.4530 \\
\hline Successful rate (\%) & - & 46 & 24 \\
\hline Run time (sec) & - & 13.3928 & 15.8138 \\
\hline
\end{tabular}

TABLE VI. COMPARISION OF SOS WITH OTHER METHODS IN THE 69NODE SYSTEM FOR CASE-1

\begin{tabular}{|c|c|c|c|}
\hline Method & Opened switches & $\Delta \boldsymbol{P}(\mathbf{k W})$ & $\boldsymbol{V}_{\min }$ (p.u.) \\
\hline SOS & $14,57,61,69,70$ & 98.5875 & 0.9495 \\
\hline CSA [17] & $14,57,61,69,70$ & 98.5875 & 0.9495 \\
\hline ASFLA [33] & $14,56,61,69,70$ & 98.59 & 0.9495 \\
\hline FWA [32] & $14,56,61,69,70$ & 98.59 & 0.9495 \\
\hline
\end{tabular}

TABLE VII. RESULTS OF CASE-2 OF THE 69-NODE SY STEM AFTER 50 INDEPENDENT RUNS

\begin{tabular}{|c|c|c|c|c|}
\hline Item & Initial & SOS & PSO & CSA [17] \\
\hline Opened switches & $\begin{array}{c}69,70, \\
71,72,73\end{array}$ & $\begin{array}{c}14,57,64, \\
69,70\end{array}$ & $\begin{array}{c}14,57,64, \\
69,70\end{array}$ & $\begin{array}{c}14,58, \\
64,69,70\end{array}$ \\
\hline $\begin{array}{c}\text { DG size in MW } \\
\text { (node) }\end{array}$ & \multicolumn{4}{|c|}{$0.6022(11) ; 0.3804(18) ; 2(61)$} \\
\hline$\Delta \boldsymbol{P}$ (kW) & 72.4343 & 41.1317 & 41.1317 & 41.13 \\
\hline $\boldsymbol{V}_{\min }$ (p.u.) & 0.9890 & 0.98283 & 0.98283 & 0.9828 \\
\hline Fit $_{\text {max }}$ & - & 42.2749 & 44.7133 & - \\
\hline Fit $_{\min }$ & - & 41.1317 & 41.1317 & - \\
\hline Fit $_{\text {average }}$ & - & 41.4486 & 43.0842 & - \\
\hline STD of fit. & - & 0.4794 & 0.8452 & - \\
\hline $\begin{array}{c}\text { Successful rate } \\
\text { (\%) }\end{array}$ & - & 42 & 4 & - \\
\hline Run time (sec) & - & 12.2278 & 14.9856 & - \\
\hline
\end{tabular}


The comparison of SOS and PSO in case-3 for the 69-node test system presented in Table VIII also leads to the similar conclusion that SOS is better than PSO in terms of successful rate, quality of fitness function values and running time. For the network with existing three type-PQ DGs with sizes of $0.0871+\mathrm{j} 0.2096,1.4155+\mathrm{j} 1.0131$ and $0.5643+\mathrm{j} 0.3856 \mathrm{MW}$ at nodes 68,61 , and 27 respectively, after reconfiguration, SOS gained the optimal configuration in 18 runs corresponding to $36 \%$ while PSO did it only in 7 runs.

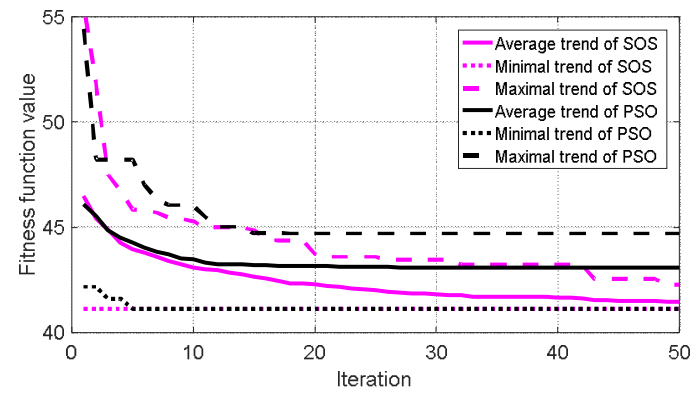

Fig. 10. Convergence trends of SOS and PSO in 50 runs for the 69-node test system in case- 2

TABLE VIII. RESULTS OF CASE-3 OF THE 69-NODE SY STEM AFTER 50 INDEPENDENT RUNS

\begin{tabular}{|c|c|c|c|c|}
\hline Item & Initial & SOS & PSO & GWO [30] \\
\hline Opened switches & $\begin{array}{c}69,70, \\
71,72, \\
73\end{array}$ & $\begin{array}{c}10,14, \\
17,56, \\
72\end{array}$ & $\begin{array}{c}10,14, \\
17,56, \\
72\end{array}$ & $\begin{array}{c}14,57,61 \\
69,70\end{array}$ \\
\hline $\begin{array}{l}\text { DG size in MVA } \\
\text { (node) }\end{array}$ & \multicolumn{4}{|c|}{$\begin{array}{c}0.0871+\mathrm{j} 0.2096(68) ; 1.4155+\mathrm{j} 1.0131(61) \\
0.5643+\mathrm{j} 0.3856(27)\end{array}$} \\
\hline$\Delta P(\mathbf{k W})$ & 15.77 & 7.6707 & 7.6707 & 8.4784 \\
\hline$V \min$ (p.u.) & 0.984 & 0.9934 & 0.9934 & - \\
\hline Fitmax & - & 7.9488 & 8.7164 & - \\
\hline Fitmin & - & 7.6707 & 7.6707 & - \\
\hline Fitaverage & - & 7.7247 & 7.9065 & - \\
\hline STD of fit & - & 0.0840 & 0.24383 & - \\
\hline Successful rate (\%) & - & 36 & 14 & - \\
\hline Run time (sec) & - & 10.7863 & 14.24 & - \\
\hline
\end{tabular}

Maximal, average and STD of the fitness function values obtained by SOS were lower than those of PSO. Moreover the running time of SOS was also lower. The average, minimal and maximal convergence trends of PSO and the proposed SOS in 50 runs for case- 3 are shown in Figure 11. The power loss of the optimal configuration obtained by SOS was $0.8077 \mathrm{~kW}$ lower than GWO [30].

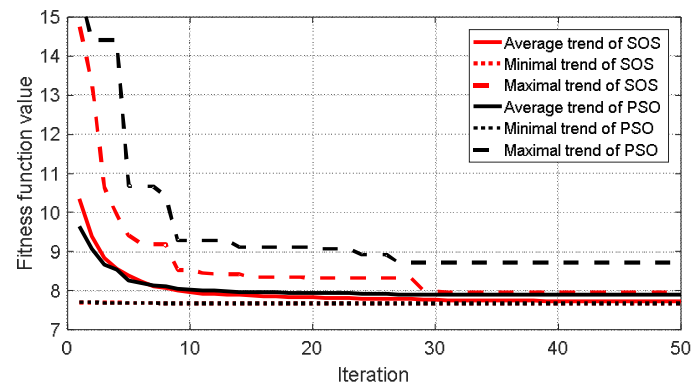

Fig. 11. Convergence trends of SOS and PSO in 50 runs for the 69-node test system in case-3
The voltage profile of the 69-node system achieved by the proposed SOS technique is shown in Figure 12. In the initial configuration there were many nodes with lower voltage magnitude than the permissible range, such as nodes 57-69. Most of them improved after reconfiguration by SOS in case- 1 . Only the node 61 had voltage magnitude of 0.9495 which was a bit lower than the lower permissible limit. In case- 2 and case3 , the voltage magnitude of all nodes was within the permissible range.

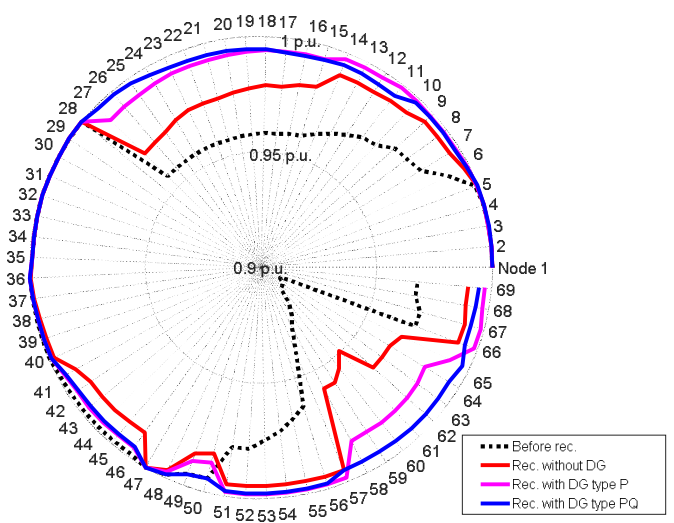

Fig. 12. Voltage profile of the 69-node test system before and after performing reconfiguration

\section{CONCLUSION}

This paper presents a method of network reconfiguration based on SOS for finding the optimal electric network configuration with minimal power loss. The effectiveness of the proposed SOS method is evaluated on a 33-node, and a 69node test system. Three cases of performing network reconfiguration were considered: on a system without DG placement, a system with installed type-P DGs, and a system with installed type-PQ DGs. The numerical results in 50 independent runs show that SOS outperformed PSO in terms of successful rate, quality of the obtained optimal solution, number of convergence iterations, and running time. The numerical results also demonstrate that SOS is better than some other techniques available in the literature. Furthermore, the successful applying of SOS in the network reconfiguration problem with respect to reducing active power loss in the three test systems showed the potential of using SOS to satisfy other objective functions without turning any control parameters during the applying process.

\section{REFERENCES}

[1] W. L. Theo, J. S. Lim, W. S. Ho, H. Hashim, C. T. Lee, "Review of distributed generation (DG) system planning and optimisation techniques: Comparison of numerical and mathematical modelling methods", Renewable and Sustainable Energy Reviews, Vol. 67, pp. 531-573, 2017

[2] Y. Mobarak, A. Hemeida, A. El-Bahnasawy, M. Hamada, "Reactive power compensation on Egypt Electricity Network for optimal energy saving", Engineering, Technology \& Applied Science Research, Vol. 9, No. 1, pp. 3699-3704, 2019

[3] P. Balamurugan, "Optimal allocation of DSTATCOM in distribution network using hale Optimization Algorithm", Engineering, Technology \& Applied Science Research, Vol. 8, No. 5, pp. 3445-3449, 2018 
[4] Z. Masomi Zohrabad, "Application of Hybrid HS and Tabu Search Algorithm for optimal location of FACTS devices to reduce power losses in power systems", Engineering, Technology \& Applied Science Research, Vol. 6, No. 6, pp. 1217-1220, 2016

[5] A. Merlin, H. Back, "Search for a minimal loss operating spanning tree configuration in an urban power distribution system", 5th Power System Computation Conference, Cambridge, UK, September 1-5, 1975

[6] D. Shirmohammadi, H. W. Hong, "Reconfiguration of electric distribution networks for resistive line losses reduction", IEEE Transactions on Power Delivery, Vol. 4, No. 2, pp. 1492-1498, 1989

[7] S. Civanlar, J. J. Grainger, H. Yin, S. S. H. Lee, "Distribution feeder reconfiguration for loss reduction", IEEE Transactions on Power Delivery, Vol. 3, No. 3, pp. 1217-1223, 1988

[8] D. L. Duan, X. D. Ling, X. Y. Wu, B. Zhong, "Reconfiguration of distribution network for loss reduction and reliability improvement based on an enhanced genetic algorithm", International Journal of Electrical Power and Energy Systems, Vol. 64, pp. 88-95, 2015

[9] K. K. Kumar, N. Venkata, S. Kamakshaiah, "FDR particle swarm algorithm for network reconfiguration of distribution systems", Journal of Theoretical and Applied Information Technology, Vol. 36, No. 2, pp. 174-181, 2012

[10] T. M. Khalil, A. V Gorpinich, "Reconfiguration for loss reduction of distribution systems using selective Particle Swarm Optimization", International Journal of Multidisciplinary Sciences and Engineering, Vol. 3, No. 6, pp. 16-21, 2012

[11] A. Y. Abdelaziz, S. F. Mekhamer, F. M. Mohammed, M. A. L. Badr, "A modified Particle Swarm technique for distribution systems reconfiguration", The Online Journal on Electronics and Electrical Engineering, Vol. 1, No. 1, pp. 121-129, 2009

[12] E. Mahboubi-Moghaddam, M. R. Narimani, M. H. Khooban, A. Azizivahed, M. Javid Sharifi, "Multi-objective distribution feeder reconfiguration to improve transient stability, and minimize power loss and operation cost using an enhanced evolutionary algorithm at the presence of distributed generations", International Journal of Electrical Power \& Energy Systems, Vol. 76, pp. 35-43, 2016

[13] A. Mohamed Imran, M. Kowsalya, "A new power system reconfiguration scheme for power loss minimization and voltage profile enhancement using fireworks algorithm", International Journal of Electrical Power \& Energy Systems, Vol. 62, pp. 312-322, 2014

[14] T. T. Nguyen, T. T. Nguyen, A. V. Truong, Q. T. Nguyen, T. A. Phung, "Multi-objective electric distribution network reconfiguration solution using runner-root algorithm", Applied Soft Computing, Vol. 52, pp. 93108,2017

[15] A. V. Truong, T. N. Ton, T. T. Nguyen, T. L. Duong, "Two states for optimal position and capacity of distributed generators considering network reconfiguration for power loss minimization based on runner root algorithm”, Energies, Vol. 12, No. 1, Article ID 106, 2019

[16] T. T. Nguyen, A. V. Truong, "Distribution network reconfiguration for power loss minimization and voltage profile improvement using cuckoo search algorithm", International Journal of Electrical Power \& Energy Systems, Vol. 68, pp. 233-242, 2015

[17] T. T. Nguyen, A. V. Truong, T. A. Phung, "A novel method based on adaptive cuckoo search for optimal network reconfiguration and distributed generation allocation in distribution network", International Journal of Electrical Power \& Energy Systems, Vol. 78, pp. 801-815, 2016

[18] M. Y. Cheng, D. Prayogo, "Symbiotic Organisms Search: A new metaheuristic optimization algorithm", Computers and Structures, Vol. 139, pp. 98-112, 2014

[19] A. E. Ezugwu, D. Prayogo, "Symbiotic Organisms Search Algorithm: theory, recent advances and applications", Expert Systems with Applications, Vol. 119, pp. 184-209, 2019

[20] S. Saha, V. Mukherjee, "Optimal placement and sizing of DGs in RDS using chaos embedded SOS algorithm", IET Generation, Transmission \& Distribution, Vol. 10, No. 14, pp. 3671-3680, 2016

[21] T. P. Nguyen, V. N. Dieu, P. Vasant, "Symbiotic Organism Search Algorithm for optimal size and siting of distributed generators in distribution systems", International Journal of Energy Optimization and Engineering, Vol. 6, No. 3, pp. 1-28, 2017

[22] P. Balachennaiah, M. Suryakalavathi, "Real power loss minimization using symbiotic organisms search algorithm", 2015 Annual IEEE India Conference, New Delhi, India, December 17-20, 2015

[23] M. P. Lalitha, P. S. Babu, B. Adivesh, "Optimal distributed generation and capacitor placement for loss minimization and voltage profile improvement using Symbiotic Organisms Search Algorithm", International Journal of Electrical Engineering, Vol. 9, No. 3, pp. 249261, 2016

[24] M. Kenan Dosoglu, U. Guvenc, S. Duman, Y. Sonmez, H. Tolga Kahraman, "Symbiotic organisms search optimization algorithm for economic/emission dispatch problem in power systems", Neural Computing and Applications, Vol. 29, No. 3, pp. 721-737, 2018

[25] D. Prasad, V. Mukherjee, "A novel symbiotic organisms search algorithm for optimal power flow of power system with FACTS devices", Engineering Science and Technology, an International Journal, Vol. 19, No. 1, pp. 79-89, 2016

[26] A. T. Boum, P. R. Ndjependa, J. N. Bisse, "Optimal reconfiguration of power distribution systems based on Symbiotic Organism Search algorithm", Journal of Power and Energy Engineering, Vol. 5, No. 11, 2017

[27] A. Y. Abdelaziz, F. M. Mohamed, S. F. Mekhamer, M. A. L. Badr, "Distribution system reconfiguration using a modified Tabu Search algorithm", Electric Power Systems Research, Vol. 80, No. 8, pp. 943953, 2010

[28] M. E. Baran, F. F. Wu, "Network reconfiguration in distribution systems for loss reduction and load balancing", IEEE Transactions on Power Delivery, Vol. 4, No. 2. pp. 1401-1407, 1989

[29] R. Pegado, Z. Naupari, Y. Molina, C. Castillo, "Radial distribution network reconfiguration for power losses reduction based on improved selective BPSO”, Electric Power Systems Research, Vol. 169, pp. 206213, 2019

[30] M. F. Abd El-salam, E. Beshr, M. B. Eteiba, "A new hybrid technique for minimizing power losses in a distribution system by optimal sizing and siting of distributed generators with network reconfiguration", Energies, Vol. 11, No. 12, Article ID 3351, 2018

[31] H. D. Chiang, R. Jean-Jumeau, "Optimal network reconfigurations in distribution systems: Part 2: Solution algorithms and numerical results", IEEE Transactions on Power Delivery, Vol. 5, No. 3, pp. 1568-1574, 1990

[32] A. Mohamed Imran, M. Kowsalya, D. P. Kothari, "A novel integration technique for optimal network reconfiguration and distributed generation placement in power distribution networks", International Journal of Electrical Power and Energy Systems, Vol. 63, pp. 461-472, 2014

[33] A. Onlam, D. Yodphet, R. Chatthaworn, C. Surawanitkun, A. Siritaratiwat, P. Khunkitti, "Power loss minimization and voltage stability improvement in electrical distribution system via network reconfiguration and distributed generation placement using novel adaptive Shuffled Frogs Leaping Algorithm”, Energies, Vol. 12, No. 3, Article ID 553, 2019 